Revue d'histoire de l'Amérique française

BRS REVUE D.HISTOIRE DE L'AMÉRIQUE FRANÇAISE

\title{
Honoré Mercier, la minorité protestante du Québec et la loi relative au règlement de la question des biens des Jésuites
}

\section{J. R. Miller}

Volume 27, numéro 4, mars 1974

URI : https://id.erudit.org/iderudit/303305ar

DOI : https://doi.org/10.7202/303305ar

Aller au sommaire du numéro

Éditeur(s)

Institut d'histoire de l'Amérique française

ISSN

0035-2357 (imprimé)

1492-1383 (numérique)

Découvrir la revue

Citer cet article

Miller, J. R. (1974). Honoré Mercier, la minorité protestante du Québec et la loi relative au règlement de la question des biens des Jésuites. Revue d'histoire de l'Amérique française, 27(4), 483-507. https://doi.org/10.7202/303305ar d'utilisation que vous pouvez consulter en ligne. 


\title{
HONORE MERCIER, LA MINORITÉ PROTESTANTE DU QUÉBEC ET LA LOI RELATIVE AU RËGLEMENT DE LA QUESTION DES BIENS DES JÉSUITES
}

\author{
J. R. Miller \\ Département d'histoire \\ Université de Saskatchewan
}

"Vos travaux législatifs sont terminés, et vous allez pouvoir retourner dans vos foyers, où vous jouirez d'un repos que vous avez si bien gagné." ${ }^{1}$ Il était trois heures de l'après-midi ; c'était le 12 juillet 1888. Et, tandis qu'à travers le Canada, les Orangistes commémoraient le deuxième centenaire de la Révolution glorieuse d'Angleterre, le lieutenant-gouverneur du Québec, sir AugusteRéal Angers, prorogeait l'Assemblée législative. Au cours des derniers jours de la session, Angers avait signé un certain nombre de projets de lois. Parmi ceux-ci figurait la loi sur les biens des Jésuites.* C'était là poser un geste qui devait bientôt inciter les Orangistes à attaquer le gouvernement d'Honoré Mercier, l'Eglise catholique et les Canadiens français comme groupe ethnique. Ce 12 juillet 1888 marquait le début de deux années difficiles dans l'histoire de la province de Québec et du Canada.

\section{I}

Le premier ministre fut sans doute surpris de constater la fureur que souleva sa législation. En fait, la loi qu'il venait de faire adopter avait pour but de résoudre un vieux problème qui remontait aux capitulations de Québec et de Montréal, de 1759 et de 1760. Au lendemain de la Conquête, les nouveaux dirigeants avaient dû prendre une décision concernant le sort des communautés religieuses qui étaient bannies, en vertu des lois anglaises, de tous les territoires britanniques. De plus, quel usage allait faire le Board of Trade des riches dotations en terres

* Nous abrégeons ainsi le titre de la loi intitulée: "Acte relatif au règlement de la question des biens des Jésuites".

1 Discours du lieutenant-gouverneur Auguste-Réal Angers à la clôture de la Session législative de 1888 (12 juillet 1888), Débats de la Législature de la province de Québec. Publiés sous les auspices de la Législature de la province de Québec par Alphonse Desjardins (Québec, 1888): 1737-1738.

[ 483 ]

RHAF, vol. 27, no 4 (mars 1974) 
que la Société de Jésus avait reçues de la Couronne de France et de fervents bienfaiteurs pour lui permettre de poursuivre son œuvre d'évangélisation et d'éducation en Nouvelle-France ? Les autorités anglaises en vinrent à la conclusion qu'il fallait supprimer l'Ordre des Jésuites de la province de Québec, mais elles décidèrent en toute sagesse de n'effectuer cette suppression que graduellement et en douceur. Les Jésuites se virent donc interdire de recruter de nouveaux membres dans la province et de faire venir d'Europe des sujets déjà ordonnés de sorte que s'éteignit, quarante ans plus tard, le dernier Père survivant en terre canadienne. En vertu du droit d'aubaine, le gouvernement britannique prenait possession, en mars 1800, des riches biens des Jésuites.

Toutefois, deux autres groupes réclamèrent aussi leur droit sur ces biens. Le clergé séculier prétendait être le bénéficiaire légitime de la succession des Jésuites, étant donnée la suppression de la Compagnie par le Pape en 1773. En effet, d'après le droit canonique, quand un ordre religieux était dissous, ses propriétés foncières revenaient aux ordinaires des diocèses. Outre cette revendication du clergé, il y avait celle des laïcs qui prétendaient que leurs représentants élus avaient juridiction en cette affaire. Selon eux, les terres des Jésuites avaient été concédées non pour l'usage personnel de leurs membres, mais en vue de l'œuvre d'éducation. Comme dans les années qui suivirent la Conquête, les dirigeants du clergé s'étaient montrés hésitants à se prévaloir de leurs droits, les réclamations des citoyens constituaient l'obstacle le plus sérieux à la mainmise britannique sur les biens des Jésuites. Après 1791, les représentants du peuple exigèrent que les biens fussent réservés à l'éducation et que l'administration relevât de l'Assemblée législative. Cette lutte pour le contrôle des revenus des biens ne manqua pas, durant les premières décennies du dix-neuvième siècle, de devenir une sérieuse cause de conflit entre l'Assemblée législative et le Conseil exécutif du Bas-Canada. Finalement, de guerre lasse, en 1831, l'administration coloniale décida de remettre les biens des Jésuites aux mains de l'Assemblée législative pour qu'ils servent à l'éducation supérieure.

Dix ans plus tard, le clergé revint à la charge avec ses revendications. Ayant à leur tête l'évêque de Montréal, Mgr Ignace Bourget, les ultramontains firent pression pour qu'on restitue à l'Eglise la propriété des biens ou, à défaut, qu'on accorde une généreuse compensation en dédommagement des pertes subies. La cause de l'Eglise se trouvait alors renforcée par la présence 
des Jésuites qui, sur l'instance de Mgr Bourget, étaient revenus au Canada au cours de la décennie de 1840, après le rétablissement de la Compagnie. Ces revendications étaient reliées au projet de Mgr Bourget et de la Compagnie de Jésus d'établir une université catholique sous la direction des Jésuites à Montréal. Ce projet avait pour but de procurer aux catholiques de la région de Montréal qui ne pouvaient se rendre à Québec une éducation supérieure dispensée sous la surveillance des ultramontains.

L'Eglise en général et les ultramontains en particulier continuèrent de réclamer une compensation après que les terres eussent été cédées au gouvernement provincial lors de la Confédération. Les chances de succès du clergé étaient naturellement accrues $d u$ fait que leurs revendications s'adressaient désormais à une Assemblée législative formée d'une grande majorité de Canadiens français et catholiques. Toutefois, en dépit de ce climat favorable, le clergé ne parvint pas à obtenir gain de cause, faute d'union entre ses membres. L'Eglise catholique du Québec était de plus en plus divisée par des controverses entre libéraux et ultramontains. Les Jésuites du Collège Sainte-Marie, qui faisaient partie de ce dernier groupe dirigé par les évêques Bourget et Laflèche, avaient à faire face à l'opposition du groupe libéral mené par l'Archevêque de Québec et par l'Université Laval. Cette lutte était en outre une manifestation de la rivalité qui existait entre les villes de Québec et de Montréal. La division des esprits était si profonde que les efforts répétés de plusieurs ministères québécois, pour en arriver à une entente au sujet des indemnités et pour obtenir un titre sûr aux terres, n'aboutirent jamais. La question des biens des Jésuites mettait l'Eglise dans l'embarras et faisait obstacle au développement de la province.

Quand Honoré Mercier accéda au pouvoir en 1886, il était au courant de ces problèmes et bien décidé à les résoudre. Convaincu qu'une solution s'imposait, Mercier voyait là, en même temps, l'occasion de manifester sa reconnaissance à ses anciens maîtres et alliés politiques, les Jésuites. Comme première mesure, le premier ministre fit voter l'incorporation de la Compagnie de Jésus par la Législature en 1887. Puis il entama des pourparlers avec le Saint-Siège en vue de conclure une entente laquelle, espérait-il, se ferait surtout à l'avantage de ses amis les Jésuites. Les clauses de cette entente élaborées par le représentant du Pape, le Père Turgeon de la Compagnie de Jésus, stipulaient que la province verserait à l'Eglise, comme indemnité, la 
somme de $\$ 400,000$ et la rétrocession des droits du gouvernement sur la commune de Laprairie, située près de Montréal, en retour de la renonciation à toute revendication future. La distribution des fonds entre les divers groupes catholiques - les Jésuites, l'Université Laval et la hiérarchie - serait laissée à la discrétion du Saint-Siège. ${ }^{2}$ Mercier se rendait bien compte que si l'entente qu'il avait conclue avec Turgeon était habile, elle était aussi très périlleuse. Il y avait surtout le danger que les amis du cardinal Taschereau, irrités, attaquent publiquement le projet avec toutes les conséquences politiques désastreuses qui pourraient en résulter. Le premier ministre pouvait éviter le danger en persuadant la population que l'arrangement avait la sanction du Saint-Siège, ce qu'on pourrait rendre évident en incluant dans le projet de loi la longue correspondance entre Québec et Rome, antérieure aux négociations, et en faisant intervenir le Pape dans la distribution des fonds. Mercier justifiait l'insertion de cette correspondance dans le projet de loi en disant: "Nous voulons que la ratification soit donnée par le chef de l'Eglise, afin que tous les intéressés soient liés." Et il ajoutait: "Il ne s'agit pas de faire sanctionner la loi par le Pape. Il ne faut pas jouer sur les mots. La loi sera sanctionnée par le lieutenantgouverneur [et pour] qu'il n'y ait pas de malentendu, que la transaction soit finale, que le règlement ne puisse plus être discuté par les autorités religieuses, nous exigeons que le Pape ratifie l'arrangement." 3 Mais comment les protestants réagiraient-ils dans cette affaire ? Il y avait au moins deux objections qu'ils pouvaient soulever. Premièrement, il se pourrait bien que la minorité protestante du Québec ne comprenne pas pourquoi le préambule de ce projet de loi accordait une telle importance au Pape ou encore pourquoi, selon toute apparence, l'arrangement dépendait de son bon vouloir. De plus, comme protestants, ils pouvaient considérer ce recours à Rome comme une violation d'un de leurs plus chers principes, celui de la séparation de

2 R. C. Dalton, The Jesuits' Estates Question 1760-1888: A Study of the Background for the Agitation of 1889 (Toronto, 1968) ; J. R. Miller, "The Impact of the Jesuits' Estates Act on Canadian Politics, 1888-1891" (thèse de doctorat, Université de Toronto, 1972), 1-45; G.-E. Giguère, s.j., "Restauration de la Compagnie de Jésus au Canada", La Société canadienne d'histoire de l'Eglise catholique, 36 (Sessions d'Etude 1969) : 67-73; P. Sylvain, "Les difficiles débuts de l'Université Laval", Les Cahiers des Dix, 36 (1971) : 211-234; A. Lavallée, "La querelle universitaire québécoise, 18761889", Revue d'histoire de l'Amérique française, XXVI, no 1 (juin 1972): $67-81$.

3 J.-O. Pelland, éd., Biographie, discours, conférences etc. de l'hon. Honoré Mercier (Montréal, 1890), 389. 
l'Eglise et de l'Etat. Le premier ministre pouvait tenter de réfuter ces objections en expliquant avec soin aux protestants que le recours au Pape n'avait d'autre but que de garantir que tous les groupes catholiques accepteraient l'arrangement; mais les protestants comprendraient-ils ce raisonnement et seraient-ils prêts à l'approuver ? De toute façon, il y avait une autre raison qui pouvait porter les protestants à s'opposer au règlement: la question d'argent. En vertu de la loi de 1831, les revenus des biens des Jésuites servaient à maintenir l'éducation supérieure dans la province de Québec, tant du côté des protestants que de celui des catholiques. Mais voilà que le gouvernement du Québec proposait de s'emparer des biens pour ses propres fins en offrant de payer à l'Eglise catholique une indemnité en retour de l'abandon de toute réclamation au sujet des terres. Les protestants allaient-ils donner leur acquiescement à tout cela ?

En fait, le premier minisire Mercier avait des raisons plus sérieuses encore de se soucier de la réaction des protestants. Il savait de façon sûre que la minorité protestante était méfiante en ce qui avait trait aux Jésuites et à leurs biens. Les protestants avaient déjà manifesté leur colère lors d'une tentative antérieure de Mgr Bourget et des Jésuites pour obtenir des compensations. Un pamphlétaire anonyme avait fait cette mise en garde: "If they [les protestants] yield an iota here, the Catholics will soon, most naturally, attempt greater encroachments and infringement of rights." " Les protestants du Québec regardaient d'un œil hostile les Jésuites et leurs alliés ultramontains. Au cours de l'agitation ultramontaine des années 1870, leur colère s'était manifestée tant au sein de la Protestant Defence League, fondée par L. S. Huntington, membre du parti libéral, que par une série de pamphlets. ${ }^{5}$ Mercier ne pouvait l'oublier car son parti avait alors bénéficié de l'opposition protestante à l'influence grandissante des ultramontains et de leur réaction à "l'influence indue" qui, dans la plupart des cas, avait servi la cause des Bleus. L'habitude regrettable des ministères conservateurs de

4 A. Rankin, Jesuits' Estates of Canada: Public Property (Montréal, $1850)$ : cité par Dalton, Jesuits' Estates, 119.

5 J. S. Willison, Sir Wilfrid Laurier and the Liberal Party (Toronto, 1903), I: 262-266 et 277-282; A. T. Galt, Church and State (Montréal, 1876) ; A. T. Galt, Civil Liberty in Lower Canada (Montréal, 1876) ; A. T. Galt, $A$ protest Against the Efforts now being made in Canada by the Roman Catholic Hierarchy to put into Practice among Her Majesty's Protestant Subjects the Doctrines of the Syllabus and the Vatican (London, 1877); T. White, The Protestant Minority in Quebec in its Political Relations with the Roman Catholic Majority. A Letter addressed to Sir Alexander Tilloch Galt, K.C.M.G. (Montréal, 1876). 
s'adonner à la corruption politique avait aussi rebuté les protestants qui se piquaient de pureté de mœurs en matière politique. Par conséquent, les libéraux considéraient les protestants comme un appui politique important.

En 1886, les protestants avaient continué d'appuyer le parti libéral même si Mercier militait sous l'étiquette nationaliste, parce que, pour beaucoup d'entre eux, l'appellation libérale signifiait encore pureté politique et prudente administration des finances publiques. ${ }^{6}$ Mais bien qu'il jouissait encore de leur appui en 1886, Mercier se devait de tenir compte de leurs susceptibilités en ce qui regardait non seulement les questions religieuses mais aussi l'attribution des fonds publics. Le premier ministre avait cherché à réduire le taux d'intérêt sur la dette amortissable, à imposer à la province une taxe commerciale dans le but de réduire le poids de la dette publique et de recueillir des fonds pour son ambitieuse politique ferroviaire. Malheureusement, les protestants qui étaient à la tête de la plupart des grandes corporations du Québec pensaient que ces mesures étaient destinées à leur faire payer les frais d'une politique nationaliste canadienne-française. ${ }^{7}$ Même ceux qui n'étaient pas directement intéressés au commerce ou à la finance, notamment les agriculteurs des Cantons de l'Est, avaient le sentiment d'avoir à souffrir de cette politique. Les plus fanatiques d'entre ceux-ci, constatant l'affluence des francophones dans les Cantons de l'Est, croyaient qu'ils étaient victimes d'une conspiration nationaliste dirigée par les éléments ultramontains de l'Eglise catholique pour les déloger de leurs enclaves rurales traditionnelles. ${ }^{8}$ Et tous voyaient avec méfiance les énormes dépenses publiques du régime Mercier et prêtaient l'oreille d'abord avec incrédulité, puis avec dégoût, aux rumeurs de corruption qui allaient de pair avec ces dépenses. ${ }^{9}$ Les protestants avaient aussi des griefs concernant l'éducation. Ils étaient irrités par le refus de la Législature d'approuver le projet de loi, généralement connu sous le nom

${ }^{6}$ R. W. Cox, "The Quebec Provincial General Election of 1886", thèse de M.A. (histoire) (Université McGill, 1948), 112-128.

7 Miller, "Impact of the Jesuits' Estates Act", 71-74; J. M. Bliss, " 'Dyspepsia of the Mind': The Canadian Businessman and His Enemies, 1880-1914", D. S. MacMillan, ed., Canadian Business History: Selected Studies, 1497-1971 (Toronto, 1972), 179.

8 Equal Rights Association, Important Letter by a Resident of Quebec as to 'The Disabilities of Protestants in that Province' (Toronto, 1890), 5-7; J. I. Hunter, "The French Invasion of the Eastern Townships: A Regional Study" (thèse de M.A. (sociologie), (Université McGill, 1939).

9 Archives publiques du Canada [APC], Fonds Honoré Mercier, I, Calixte LeBeuf à E. Pacaud, 23 avril 1889. 
de "B.A.Bill",* qui visait à faire du baccalauréat le prérequis à l'admission aux études professionnelles dans la province de Québec. ${ }^{10}$ Ils étaient aussi d'avis que la répartition des taxes scolaires provenant des compagnies incorporées devait être faite selon le désir des actionnaires et non au prorata de la population protestante et catholique de la province. ${ }^{11}$ Mais la majorité catholique au Conseil de l'Instruction publique ne partageait pas ce point de vue. Toutes ces questions - la taxe commerciale, la conversion de la dette amortissable, l'émigration canadiennefrançaise vers les Cantons de l'Est, le "B.A.Bill", le refus d'une demande de majoration des revenus des taxes scolaires et les rumeurs croissantes de corruption - eurent pour résultat de détacher de plus en plus les protestants anglophones de leur vieille allégeance politique, le parti libéral. "Mercier... is regarded with very uneasy and half hearted approval, rapporte un protestant, and his difficulties with [them] are rather increasing." 12

Voilà donc les raisons pour lesquelles Mercier avait à se soucier non seulement de la réaction du cardinal Taschereau à son projet de loi concernant les biens des Jésuites, mais aussi de celle des protestants. A vrai dire, il pouvait s'enhardir du fait qu'en 1887 sa loi d'incorporation des Jésuites n'avait suscité que peu de commentaires. Les protestations isolées de la Protestant Ministerial Association à ce projet de loi avaient été timides comparativement à l'obstruction qu'avaient essayé d'apporter Mgr Taschereau et quelques autres évêques. ${ }^{13}$ Mercier aurait néanmoins à se concilier l'appui des protestants.

La solution qui s'offrit à lui fut d'inclure, dans l'arrangement, un octroi de $\$ 60,000$ en faveur du comité protestant du Conseil de l'Instruction publique, montant correspondant à la proportion de la population protestante dans la province. Le stratagème sembla réussir, bien qu'un ou deux députés protestants s'objectèrent à ce que Mercier considérât la confiscation faite par l'administration britannique en 1800 des biens des Jésuites comme un acte de "spoliation" et contestèrent le rôle du

* Projet de loi reconnaissant le diplôme de bachelier ès arts suffisant pour l'admission à l'étude des professions légale, médicale et du notariat. 10 Québec, Sessional Papers ( $N^{\circ} 5$ ) 1888, t. 22, Sommaire des procédés d'une assemblée du Comité Protestant du Conseil de l'Instruction publique, 21 sept. et 23 nov. 1887 : $265-267$.

11 Québec, Sessional Papers (No 5) 1889, t. 23, Sommaire des procédés d'une assemblée du Comité Protestant du Conseil de l'Instruction publique, 28 nov. 1888 et 6 fév. $1889: 375-376$ et 378-381.

12 APC, Fonds Sir Wilfrid Laurier, 2: 759-761: W. C. Gibson à W. Laurier, 18 sept. 1888.

13 Dalton, Jesuits' Estates, 148-155. 
Pape comme distributeur de fonds publics. ${ }^{14}$ Les Bleus tentèrent timidement d'ennuyer le gouvernement en accusant Mercier de vouloir récompenser les Jésuites pour leur appui en 1886. ${ }^{15}$ Toutefois l'opposition se refusa à entraver la promulgation de la loi et le caucus conservateur dissuada un collègue protestant impétueux de présenter un amendement. ${ }^{16}$ La faiblesse de l'opposition devint évidente lorsque Mercier insista pour obtenir l'enregistrement du vote. Les quelques députés qui s'étaient d'abord opposés au projet de loi retirèrent leur opposition et on proclama l'unanimité. ${ }^{17} \mathrm{Au}$ Conseil législatif, les protestants ne formulèrent aucune objection au projet de loi et, le 12 juillet 1888, au moment où les Orangistes célébraient l'anniversaire de la Révolution glorieuse et que l'Assemblée législative était prorogée, le lieutenant-gouverneur Angers accordait la sanction royale à la loi relative au règlement de la question des biens des Jésuites.

\section{II}

L'opposition protestante en dehors de la Législature s'avéra également faible. L'organe conservateur, la Gazette de Montréal, se contenta de dire que la loi était "a skilful party manoeuvre", mais qu'elle constituerait pour les protestants "a bitter pill"'.18 Dans les Cantons de l'Est, le Canadian Gleaner, journal qui, au dire de son propriétaire Robert Sellar, demeurait toujours au service du libéralisme sinon du parti libéral, qualifiait cette loi "an open defiance of the crown" et une farce puisque "[the] men to whom Mercier proposes to pay the money are no more the heirs of the Jesuits of 1780 [sic] than they are of the apostles" 19. Le St. John News condamnait les députés protestants qui "wagged their little tails and bleated faintly", quoiqu'ils n'eurent pas le courage d'émettre " 'an opposing Ba-a",.20 De son côté, le Stanstead Journal soulignait de façon sarcastique: "It would appear by this act of the Holy See that they assume the powers supposed to rest in the hands of the representatives of the people and Her Majesty's representative." ${ }^{21}$

14 Débats de la Législature de la Province de Québec, Deuxième session du sixième parlement, 10 (1888) : 1270-1271.

15 Ibid.: 1272-1282.

16 The Gazette, Montréal, 22 juin et 23 juin 1888.

17 Official Report of the Debates of the House of Commons, Third Session, Sixth Parliament (1889), 28: 840 .

18 The Gazette, Montréal, 13 juillet 1888.

19 Canadian Gleaner, Huntingdon, Québec, 14 juin et 12 juillet 1888.

20 St. John News; cité par Canadian Gleaner, 2 août 1888.

21 The Stanstead Journal, 23 août 1888. 
Bientôt, en effet, un ton plaintif commença à se faire entendre dans certains éditoriaux. Sellar regrettait la "strange apathy" des protestants qui refusaient de s'élever fermement contre la loi. ${ }^{22}$ Vers la mi-août 1888, Mercier était suffisamment convaincu de l'acceptation générale de sa loi pour pouvoir s'en vanter en disant, selon le Canadian Gleaner, "I have settled that question and thank God, it is settled forever." ${ }^{23}$

Cependant, tel n'était pas tout à fait le cas, car le règlement définitif dépendait du Pape. Mercier espérait que le Vatican attribuerait toute la somme, soit les quatre cent mille dollars, ou du moins la plus grande partie de cette somme, à la Compagnie de Jésus, ainsi qu'il s'en exprima au Père Turgeon qui se rendait à Rome pour plaider la cause de son Ordre. ${ }^{24}$ Mais l'Université Laval et Mgr Taschereau se mirent aussi à solliciter les faveurs du Saint-Siège. Le recteur de Laval, Mgr Paquet, partit pour Rome au début de juillet et, le mois suivant, le cardinal Taschereau s'efforçait de renforcer la position de Mgr Paquet au Vatican en y envoyant le juge Georges Baby, un vieil ami de l'Université. Malgré ses hésitations à entreprendre ce voyage, Baby y consentit finalement parce que "l'Université traverse en ce moment une grande crise et [que] ses amis ont de graves craintes pour son avenir". ${ }^{25}$ Comme ils l'avaient fait lorsqu'il s'était agi d'obtenir le chapeau cardinalice pour Mgr Taschereau en $1885,{ }^{26}$ les amis de Laval se tournèrent du côté d'Ottawa et du parti conservateur pour obtenir des appuis en Angleterre et à Rome. Sur les instances de Caron, Langevin et Thompson, le premier ministre Macdonald pourvut Baby de lettres de recommandation auprès de nobles catholiques influents d'Angleterre, dans l'espoir de gagner à son parti les bonnes grâces du cardinal Taschereau. ${ }^{27}$ Mais même avec l'appui du gouvernement fédéral,

22 Canadian Gleaner, 23 juin et 12 sept. 1888.

23 Ibid., 5 sept. 1888.

24 R. Rumilly, Histoire de la Province de Québec, VI: Les Nationaux (Montréal, 1941): 28 .

25 APC, Fonds Sir Adolphe Caron, 125: paquet 12627, G. Baby à Caron, 16 août 1888 .

26 D. C. Lyne, "Sir John A. Macdonald and the Appointment of Canada's First Cardinal", Revue d'études canadiennes/Journal of Canadian Studies, II, no 4 (nov. 1967) : 58-60.

27 APC, Fonds Sir John A. Macdonald, 201: 84975-84977, Sir. A. Caron à Macdonald, ? août 1888; ibid.: 84988, (copie) Caron à Macdonald, 19 août 1888; Fonds Caron, 125: paquet 12627, Sir J. A. Macdonald à Caron, 18 août 1888; ibid., Macdonald à Caron, 21 août 1888. - Voir aussi Archives de la Société de Jésus du Canada français [ASJCF], paquet 1348, A. A. Blais à [A. E. Jones, s.j.], 25 août 1888. 
Baby vit ses efforts quelque peu entravés par ses rivaux, tant à Londres qu'à Rome. Dans la capitale anglaise, il crut discerner un complot monté par les Jésuites pour le priver de l'assistance du cardinal Manning ${ }^{28}$ et, dans la Ville éternelle, il se rendit compte qu'il lui faudrait lutter non seulement contre le Père Turgeon, mais encore contre le Père Paradis des Oblats. ${ }^{29}$ Baby trouva chez les Jésuites des adversaires de taille car "MM. les $\mathrm{J}$ [ésuites] vont remuer ciel et terre, afin d'arriver au but qu'ils se proposent et ils comptent beaucoup plus aujourd'hui auprès du St Siège, par de singulières circonstances, qu'ils ne l'ont fait en aucun temps depuis l'avènement du Pape actuel." 30 Baby travailla ferme à Rome pendant plusieurs mois, s'efforçant de faire contrepoids à l'influence des Jésuites; en vérité, le SaintPère devait être excédé de tous les tracas que lui causait l'Eglise catholique au Québec.

Si les catholiques de Rome causaient des soucis au Pape, les protestants du Québec, de leur côté, en causaient à Honoré Mercier. Dans un discours prononcé à l'île d'Orléans, le premier ministre avait affirmé qu'il était nationaliste avant tout et que ce n'était qu'en second lieu qu'il était libéral. Sellar du Canadian Gleaner fit remarquer que ce que Mercier entendait par nationaliste c'était "not a man true to Canada or to the Empire, but a man who labors to convert Quebec into a purely French nationality". ${ }^{31}$ Mercier devait encore faire face au risque de désaveu par le gouvernement fédéral de la loi concernant les magistrats de district. Il réagit en fomentant de l'agitation contre Ottawa. ${ }^{32}$ Les dénonciations de Mercier à l'égard du gouvernement fédéral, ses professions de foi au nationalisme canadien-français et ses mesures législatives telles que la conversion de la dette amortissable et la loi sur les biens des Jésuites devinrent l'objet de critiques répétées dans les éditoriaux de certains journaux anglais. Le Guardian de Richmond, par exemple, dénonçait Mercier pour avoir "made the confusion of Dominion politics with local more intense than ever", et pour avoir "widened the breach" entre les "different nationalities and creeds", en augmentant le nombre de magistrats, et en ne remplissant pas ses promesses de mettre fin à la corruption dans l'attribution des

28 Fonds Macdonald, 327: 147974-147976, G. Baby à Macdonald, 17 sept. 1888; Fonds Caron, 125: paquet 12627, G. Baby à Caron, 19 sept. 1888. $29 \mathrm{Mgr}$ Duhamel assistait aussi à Rome, au nom de Taschereau.

30 Fonds Caron, 125: paquet 12627, G. Baby à Caron, 19 sept. 1888. 1888.

31 Canadian Gleaner, 23 août 1888; The Gazette, Montréal, 23 août

32 Miller, "Impact of the Jesuits' Estates Act", 72-73. 
concessions forestières. ${ }^{33}$ Comme le démontrait cette diatribe, la minorité anglophone du Québec considérait les questions de "race", de croyance, d'honnêteté politique et de taxation comme des problèmes connexes. De cet ensemble de griefs, la loi sur les biens des Jésuites émergea comme la principale source de mécontentement. Les membres du consistoire de Montréal * de l'Eglise presbytérienne s'en prenaient à la loi parce que, selon eux, elle constituait une violation du principe de la séparation de l'Eglise et de l'Etat et parce qu'elle attribuait de l'argent à "une compagnie qui s'est montrée l'ennemie de la liberté religieuse et civile dans le monde entier". ${ }^{34}$ Peu de temps après, un groupe encore plus nombreux de protestants, l'assemblée de fondation de l'Evangelical Alliance for the Dominion of Canada, qualifiait la situation de "aggressive Romanism" et demandait au gouverneur général de désavouer la loi sur les biens des Jésuites. ${ }^{35}$

Le cabinet fédéral commença alors à subir des pressions. Tandis que d'une part l'Evangelical Alliance réclamait le rejet de la loi, les Jésuites, pour leur part, pressaient le gouvernement de ne pas y opposer son veto. Le supérieur des Jésuites au Canada expliqua au ministre de la Justice que le Pape voulait savoir "if the Jesuits' Bill of indemnity runs any risk of being disallowed by the Federal Government", car le Souverain Pontife ne voulait pas rendre publique sa décision relative au partage des $\$ 400,000$ au risque de la voir aussitôt rendue "a nullity by a subsequent disallowance". ${ }^{36}$ Sur l'ordre du Préfet de la Propagande, Mercier avisa à son tour le lieutenant-gouverneur d'écrire à Ottawa que "for urgent reasons" la Province désirait "to know without delay, the intention of the advisers of his Excellency the Governor General, as to the exercise of the right of disallowance with reference to this Act". ${ }^{37}$ Et pour ajouter aux pressions exercées sur le gouvernement, on apprit que la

33 Richmond Guardian; cité par The Gazette, Montréal, 13 sept. 1888.

* Traduction de Montreal Presbytery.

34 Documents de la Session, $\mathrm{n}^{\circ} 54$ (1889), XXII, no 15: 25.

35 Archives de la Province d'Ontario [APO], Toronto Evangelical Alliance, Minute Book, Sommaire des procédés, 11 mars 1889, 8-9; The Herald, Montréal, 23 au 26 oct. 1888; Vital Questions. The Discussions of the General Christian Conference held in Montreal, Que., Canada (Montréal, 1889), 112-144 et 284-287.

36 APC, Fonds Sir John Thompson, 76: 8496, P. Hamel à Thompson, 16 octobre 1888 .

37 A. R. Angers au Secrétaire d'Etat, 15 oct. 1888; dans W. E. Hodgins, ed., Correspondence, Reports of the Ministers of Justice and Orders in Council upon the Subject of Dominion and Provincial Legislation 1867-1895 (Ottawa, 1896), 386. 
mission du juge Baby à Rome en faveur de Laval était entravée par l'impossibilité où il se trouvait d'assurer le Vatican qu'Ottawa n'opposerait pas son veto à la loi. . $^{38}$

Le Cabinet n'était pas favorable au désaveu de cette loi pour des raisons constitutionnelles, ${ }^{39}$ et, même s'il l'avait été, il n'aurait pas osé le faire pour des raisons politiques. Macdonald était persuadé que l'attitude de Mercier visait à provoquer un recours au veto. ${ }^{40}$ D'ailleurs, lors de l'agitation qui avait suivi le rejet de la loi sur les Magistrats de District, il eut un avant-goût de ce qu'il pouvait attendre des libéraux québécois s'il annulait d'autres mesures législatives provinciales. Il reçut un autre avertissement, cette fois de la Ville de Québec, quand débuta la session de janvier 1889. Le discours du Trône faisait remarquer notamment que le gouvernement fédéral "had under consideration the wisdom of disallowing the act of last session confirming the settlement made in connection with the property known as the Jesuits' estates", et que, par conséquent, "the carrying out of the principal provisions of that act has remained and will remain suspended." ${ }^{41}$ Enfin, lors d'une campagne précédant l'élection complémentaire de Laprairie pour le fédéral, les libéraux accusèrent les Tories de vouloir combattre la loi, et bien que ces accusations fussent vigoureusement déniées par la presse bleue, elles contribuèrent néanmoins à la défaite du candidat conservateur. ${ }^{42} \mathrm{~A}$ la mi-janvier, le Cabinet fédéral tenta de prévenir toute agitation politique en prenant la décision extraordinaire de faire savoir publiquement que la loi ne serait pas désavouée parce que le "subject-matter... is one of provincial concern only, having relation to a fiscal matter entirely within the control of the legislature of Quebec".43

Cette déclaration n'imposa pas le silence aux adversaires. La Protestant Ministerial Association de Montréal soutenait que cette loi était anticonstitutionnelle, non seulement à cause du rôle qu'y jouait le Pape, mais encore parce que cette mesure

38 Fonds Macdonald, 284: 130587, Sir C. Tupper à Macdonald, 19 déc. 1888; ibid.: 130602, Tupper à Macdonald, 4 janv. 1889.

39 Miller, "Impact of the Jesuits' Estates Act", 79-80.

40 J. Pope, ed., Correspondence of Sir John Macdonald: Selections from the Correspondence of the Right Honourable Sir John Alexander Macdonald, G.C.B., First Prime Minister of the Dominion of Canada (Toronto, 1921), 441; J. Pope, The Day of Sir John Macdonald (Toronto, 1915), 163-164.

41 (Rock Island, P.Q.) The Stanstead Journal, 17 janv. 1889.

42 Miller, "Impact of the Jesuits' Estates Act", 83.

43 Hodgins, Correspondence, Reports, 386. 
était préjudiciable aux droits de la minorité religieuse du Québec en matière d'éducation. Puisqu'elle mettait les revenus des biens à la disposition de la province pour tout usage de son choix alors que, par le passé, les terres avaient fait partie d'un fonds destiné à l'éducation supérieure, cette législation allait, à toute fin pratique, "to deprive the Province of Quebec of the entire proceeds of the estates as a fund for public education". Les pasteurs décidèrent d'envoyer une pétition au gouverneur général pour solliciter son appui et ils firent appel à l'aide des laïcs protestants, tant du Québec que de l'Ontario. ${ }^{44}$ Durant le mois de janvier 1889, divers événements vinrent augmenter l'indignation des protestants. Ceux-ci s'irritaient de l'attitude de certains catholiques qui semblaient croire que la Législature avait rendu un grand service à la minorité protestante en votant cette législation. Un député protestant songea à présenter un projet de loi qui aurait eu pour effet de supprimer la clause prévoyant l'octroi de $\$ 60,000$ consenti au Comité protestant du Conseil de l'Instruction publique. "Our people do not seem to be happy over it, affirmait-il, and Mr. Mercier and his friends are constantly reminding us of their generosity in this respect." ${ }^{45}$ Une autre source de mécontentement pour les protestants fut la défaite du "B.A.Bill" au Conseil législatif, défaite occasionnée par le refus du cabinet provincial d'y donner son entier appui. ${ }^{46}$ Enfin, Mercier rejeta une pétition de la Commission scolaire protestante en vue d'obtenir un réajustement du partage des taxes scolaires imposées aux compagnies incorporées. ${ }^{47}$ Cette série d'actions aviva les craintes des protestants et souleva une vague d'agitation contre la loi sur les biens des Jésuites.

La colère des protestants du Québec ne fit que s'accroître, atteignant son point culminant durant le débat que suscita cette législation provinciale à la Chambre des Communes, à la fin de mars 1889. Plus tôt, au cours du mois, le comité exécutif de l'Evangelical Alliance se réunit à Montréal et décida d'envoyer

44 The Herald, Montréal, 29 janv. 1889; The Globe, Toronto, 12 fév. 1889 ; Principal MacVicar au Presbyterian Witness, XLII, no 10 (9 mars $1889)$.

45 Université McGill, Rare Books Room, Fonds Dawson [Fonds Dawson (RBR) ], boîte "Letters 1887-1889", enveloppe "Letters 1889", W. W. Lynch à W. Dawson, 12 janv. 1889.

46 Ibid., Joly de Lotbinière à W. Dawson, s.d. [1889]; Débats de la Législature de la Province de Québec (1889), I: 235 et II : 2377-2406, 25792585.

47The Gazette, Montréal, 6 fév. 1889; Christian Guardian, 13 fév. 1889, 105; ibid., 6 mars 1889, 163. 
au gouverneur général une pétition exigeant la protection des droits scolaires des minorités religieuses, en vertu de l'article 93 de l'Acte de l'Amérique du Nord britannique. L'Alliance reprenait l'argument que la Ministerial Association avait mis de l'avant en janvier, à savoir que la loi abolissait le "trust fund" de 1831 et, par le fait même, portait préjudice aux protestants. ${ }^{48}$ La démarche de l'Alliance avait son importance car c'était le premier signe tangible d'action concertée entre les protestants du Québec qui se croyaient persécutés et leurs nombreux coreligionnaires de l'Ontario. Les protestants du Québec qui, jusquelà, s'étaient contentés de "fold their arms and like the Easterners, accept matters as fate", commençaient à s'enhardir. ${ }^{49}$ Selon un journaliste le "Riel episode did much to awaken the people of the other provinces to the danger that lurked in Quebec" et le "Jesuit bill has finished the task". Par conséquent, le "spirit of hopelessness, which has discouraged efforts at self-assertion of our rights in the past, ought to be abandoned in the prospect of assistance from Ontario and the other provinces of the Dominion to assimilate the institutions of Quebec with the spirit of the constitution". ${ }^{50}$ On proclama publiquement l'union des protestants de l'Ontario et du Québec au cours du débat à la Chambre des Communes où les députés ontariens défendirent vigoureusement les droits de la minorité du Québec. Dans l'ensemble, les députés protestants du Québec se seraient contentés de laisser les autres membres du Parlement plaider leur cause; C. W. Colby ainsi que Peter Mitchell allèrent même jusqu'à soutenir que les protestants du Québec n'avaient aucun grief à apporter. ${ }^{51} \mathrm{La}$ Protestant Ministerial Association réfuta avec colère ces assertions en apportant comme preuve les pétitions signées par six mille protestataires du Québec ainsi qu'une liste de griefs qui allaient de la répartition des revenus affectés à l'éducation jusqu'à l'expansion du système des écoles paroissiales de l'Eglise catholique. Et la Protestant Ministerial Association concluait: “... Nous déclarons de plus que les protestants de la province de Québec ne veulent rien devoir à la générosité ni à la libéralité des catholiques romains leurs compatriotes, mais qu'ils demandent simplement justice et leurs droits égaux comme sujets de la Reine." 52 Julius Scriver, député libéral de Huntingdon, se

48 Ibid., 2 mars 1889; ibid., 3 avril 1889.

49 The Globe, Toronto, 16 fév. 1889; APC, Fonds D'Alton McCarthy, 154-155, A. McGoun à D'A. McCarthy, 8 avril 1889 .

50 Canadian Gleaner, 23 fév. 1889.

51 Commons Debates (1889), I: 836-841.

52 Compte rendu officiel des Débats de la Chambre des Communes du Canada (1889), XXVIII: 914. 
prononça aussi contre la loi. Il avouait que ce qui inquiétait les protestants, c'était moins "la législation en elle-même [que leur croyance que le gouvernement soit] disposé à donner de l'influence au clergé et presque la haute main sur la législation, ce qui a créé un sentiment de malaise chez les protestants en général; ils croient, en outre, que si l'on n'a pas déjà empiété sur leurs droits, il est à craindre qu'à l'avenir l'on ne viole des principes qui leur sont chers." ${ }_{33}$ Toutefois, ni les arguments de Scriver ni ceux de la Ministerial Association n'eurent beaucoup de poids et une proposition en faveur du rejet de la loi fut battue par un vote de 188 contre 13.

La décision du Parlement alluma une flambée de protestations. La Montreal Ministerial Association se mit à alerter la population en envoyant des pasteurs dans les régions rurales, ${ }^{54}$ et elle se ligua avec l'association-sour de l'Ontario pour adopter un plan d'action concertée, persuadée qu'elle était que le "whole Dominion is compromised by this measure" ${ }^{55}$. Lorsque l'Ontario commença à tenir des réunions de protestations pour tenter de forcer le gouvernement fédéral à annuler cette loi dans le délai statutaire d'une année, soit avant le 8 août 1889, les protestants montréalais emboîtèrent le pas. A une réunion de plus de trois mille personnes, tenue à Montréal, le 25 avril, les têtes s'échauffèrent au point que l'un des orateurs alla jusqu'à refuser de reconnaître que le Québec était "a French province", insistant plutôt pour dire "that it was a British Province and that they were going to show that it was such". ${ }^{56}$ Quand à Toronto l'on forma un comité de citoyens non-engagés pour condamner l'agitation contre la loi, les protestants de Montréal réagirent par la création du Montreal Citizens' Committee, composée de cinquante-deux membres qui promirent "to take such measures to secure the disallowance of the Jesuit Estates Act; to test the constitutionality of said Act; and of the Act of Incorporation of the Jesuit Order; and to this end to co-operate with other similar committees throughout the Dominion".57 Lorsque le Toronto Citizens' Committee fit parvenir une invitation à toutes les communautés protestantes de l'Ontario d'envoyer des délégués à une convention devant se tenir à Toronto en juin, le Montreal Committee s'empressa d'en nommer pour y assis-

53 Ibid.: 916-917.

54 The Herald, Montréal, 9 avril 1889. 1889.

55 Secrétaire de l'Alliance évangélique à The Gazette, Montréal, 7 mai

56 The Herald, Montréal, 26 avril 1889.

57 Ibid. 
ter. Cette convention anti-jésuites eut pour effet de détruire l'union qui était à se former entre les mouvements de contestation du Québec et ceux de l'Ontario; les protestants du Québec en furent profondément ébranlés. ${ }^{58}$ L'une des premières mesures prises par cette assemblée fut de déclarer que cette union était l'affaire des seuls Ontariens. En réponse aux protestations vociférantes d'un groupe de protestants montréalais, les Ontariens décidèrent que les délégués des autres provinces pourraient prendre la parole et faire des propositions au cours des délibérations, mais qu'ils n'auraient pas droit de vote. De plus, l'organisation que la convention avait mise sur pied pour combattre le nationalisme canadien-français, l'Equal Rights Association de la province d'Ontario, ne prévoyait pas la participation des protestants du Québec. En fait, les délégués de Montréal et des Cantons de l'Est auraient voulu n'avoir rien à voir avec la nouvelle ERA, car son programme mettait en danger les droits des protestants du Québec. Non contents de réclamer le rejet de cette loi, les Ontariens allaient jusqu'à exiger qu'on imposât des restrictions sévères à l'usage du français dans les écoles publiques de l'Ontario et à suggérer que l'on exerçât une plus grande vigilance sur l'administration des écoles catholiques dans leur province..$^{59}$ Le docteur Davidson, un anglican de Montréal, leur fit remarquer que tout changement dans le domaine de l'éducation en Ontario pouvait mettre en péril la minorité du Québec. Et de son côté, le principal MacVicar, du Presbyterian College, fit aussi une mise en garde contre l'abolition des écoles séparées en Ontario qui pourrait entraîner le gouvernement du Québec à faire de même pour le système protestant du Québec: "[We] must do what we can rather than what we would like", déclara-t-il. ${ }^{60}$ On ne fit aucun cas des avertissements de Davidson, de MacVicar et des délégués québécois.

\section{III}

Cependant au lendemain de la convention anti-jésuites, il y eut une réconciliation entre les protestants du Québec et ceux de l'Ontario en vue de tenter un suprême effort pour amener le gouverneur général à révoquer la loi sur les biens des Jésuites. Des délégués de l'Ontario et de Montréal se rendirent en vain à Québec dans le but de persuader Lord Stanley d'exercer la

58 J. R. Miller, “Equal Rights for All': the E.R.A. and the 1890 Ontario Election", Ontario History, LXV, 4 (déc. 1973) : 214-17. 13 juin 1889 .

59 The Globe, Toronto, 12 et 13 juin 1889; Toronto Daily Mail, 12 et 60 The Globe, Toronto, 13 juin 1889. 
prérogative royale et d'imposer son veto à la loi. ${ }^{61}$ En dépit de cet échec, les protestants du Québec n'en décidèrent pas moins que "the agitation will be continued in a more extended form" ${ }^{62}$, et l'éditeur du Star de Montréal chercha vainement à convaincre le cabinet fédéral de porter la cause devant les tribunaux. ${ }^{63}$ Cependant cet ultime effort échoua, et la campagne menée par les protestants du Québec n'aboutit à rien avant le 8 août, date limite après laquelle il n'était plus constitutionnellement possible d'opposer un veto à cette loi.

Rebutés par Lord Stanley, les protestants du Québec se voyaient de plus menacés par Mercier. Dans un discours qu'il prononça à l'occasion de la Saint-Jean-Baptiste, le premier ministre évoqua l'agitation de l'Equal Rights Association pour convaincre son auditoire que les Canadiens français devaient se ranger à sa suite. "Nos ennemis sont unis dans leur haine de la patrie française; et nous, nous sommes divisés dans notre amour de cette chère patrie", déclara-t-il. "Cessons nos luttes fatricides [sic] et unissons-nous!" Et, selon le Toronto Daily Mail, il avertit aussi les protestants du Québec que "if the French Canadians were to accept the provocations that had been offered them they might not be the first victims of this agitation". De toute façon, proclama-t-il, "cette province de Québec est catholique et française, et elle restera catholique et française". ${ }^{64}$

La mise en garde de Mercier modifia l'attitude des protestants du Québec mais n'étouffa pas leur mouvement de protestation. Une filiale de l'Equal Rights Association se forma à Montréal et on mit sur pied une organisation provinciale. ${ }^{65}$ La Montreal Equal Rights Association réussit à réunir une immense foule pour écouter D'Alton McCarthy dénoncer le parti libéral et, par la même occasion, la fameuse loi. ${ }^{66}$ Concurremment, dans les Cantons de l'Est, Robert Sellar entreprenait une autre phase de sa campagne de harcèlement contre Mercier et

61 Fonds MacDonald, 94: 36633-36634, Lord Stanley à Macdonald, 2 août 1889; Morning Chronicle, Québec, Lord Stanley à Lord Knutsford, 8 août 1889, cité par Hodgins, Correspondence, Reports, 398-401.

62 The Gazette, Montréal, 3 août 1889.

$63 \mathrm{H}$. Graham au Secrétaire d'Etat, 10 juin 1889, dans Hodgins, Correspondence, Reports, 419-420; J. P. Heisler, "Sir John Thompson, 18441894" (thèse de doctorat, Université de Toronto, 1955), 190-191; Fonds Thompson, 90: 10236, Macdonald à Thompson, 24 juillet 1889; ibid., 91: 10288, Macdonald à Thompson, 2 août 1889.

${ }_{64}$ R. Rumilly, Mercier (Montréal, 1936), 363-365; Toronto Daily Mail, 25 juin 1889

65 The Herald, Montréal, 23 sept. 1889.

66 Ibid., 10 oct. 1889; The Gazette, Montréal, 9 nov. 1889. 
le parti nationaliste. ${ }^{67}$ Tandis que la vague d'agitation populaire battait son plein, le comité protestant du Conseil de l'Instruction publique entreprenait une croisade pour le redressement de ses griefs. Ce comité qui avait soigneusement étudié le texte de la loi depuis le mois de février 1889 fit savoir qu'il considérait cette législation comme étant anticonstitutionnelle parce qu'elle disposait des fonds qui avaient été réservés à l'éducation supérieure, violant ainsi le "trust" de 1831. En conséquence, les protestants exigeaient que l'on rétablisse "the cancelled trust", et demandaient, de plus, que soient levées les restrictions imposées par la loi concernant les $\$ 60,000$ accordés aux protestants pour fins d'éducation. ${ }^{68}$ Le comité protestant fit connaître sa position au cabinet fédéral, sans doute dans l'intention d'amener le Parlement à un redressement de la situation si la chose s'avérait nécessaire un jour. ${ }^{69}$ Le principal Dawson, de l'Université McGill, protesta privément auprès de conservateurs bien en vue en disant que même si sir John Thompson "may and no doubt does believe in the paramount authority of the Pope", une telle croyance "surely cannot legalise robbery". ${ }^{70}$ A l'automne, le comité protestant intensifia ses réclamations auprès du gouvernement provincial. Il se plaignait du fait que les $\$ 60,000$ accordés aux protestants étaient insuffisants car d'après la population de la province, ils avaient droit à une part plus grande. ${ }^{71} \mathrm{Et}$, au cas où Québec ferait la sourde oreille à leurs réclamations, le comité protestant envoya au Conseil privé d'Ottawa une pétition sollicitant une audience en vue de faire valoir sa cause au sujet des fonds du soi-disant "cancelled trust". ${ }^{72}$

Mercier était désormais désireux d'apaiser les protestants et de liquider la question des biens des Jésuites une fois pour toutes. Le cas des catholiques qui réclamaient des compensations avait été réglé le 5 novembre 1889 lorsque le premier ministre avait distribué les $\$ 400,000$ selon le décret du Pape, comme suit: $\$ 160,000$ aux Jésuites, $\$ 140,000$ à Laval, $\$ 10,000$

67 R. Sellar à Honoré Mercier, 2 nov. 1889, dans Toronto Daily Mail, 4 nov. 1889; voir aussi Association des Droits égaux, Letters Relative to the Rights and Present Position of the Quebec Minority (s.p., s.d. [1890]). 68 Québec, Sessional Papers (No 5) 1889, 23, Sommaire des procédés, 6 fév. 1889: 381; ibid., Sommaire des procédés, 14 mai 1889: 390-394. juin 1889

69 Fonds Macdonald, 474: 236025-236028, W. Dawson à Macdonald, 12

70 Ibid., 475: 236501, R. White à Macdonald, 15 juillet 1889, avec: 236503-236504, W. Dawson à White, s.d.

71 Québec, Sessional Papers ( $\left.N^{\circ} 5\right)$ 1890, 24, Sommaire des procédés, 25 sept. $1889: 338$.

72 The Gazette, Montréal, 28 sept. 1889. 
à chacun des six diocèses et aux deux archidiocèses, et enfin $\$ 20,000$ à la préfecture apostolique du Golfe Saint-Laurent. ${ }^{73}$ Avant de se rendre aux revendications du comité protestant Mercier insista pour que ce dernier acceptât la somme qui lui avait été adjugée, et cela, en dépit du fait que plusieurs groupes protestants incitaient le comité à refuser ce qu'ils appelaient "un pot de vin". ${ }^{74}$ Après avoir obtenu de la part du comité une promesse de coopération, Mercier s'attaqua aux griefs des protestants. Il expliqua que le gouvernement n'avait jamais eu l'intention de changer la destination des fonds affectés par la loi sur les biens des Jésuites à la promotion de l'éducation supérieure. Cependant, il ne s'opposait aucunement à l'idée de clarifier ce point par une mesure législative si le comité protestant le désirait. Il justifia la décision qu'il avait prise de ne laisser au comité que l'usage des intérêts annuels sur son octroi de $\$ 60,000$, en faisant remarquer que cette clause avait été introduite à la demande expresse des membres protestants de l'Assemblée législative. Le gouvernement était néanmoins prêt, avec l'appui des députés protestants, à modifier la loi de façon à ce que l'octroi fût affranchi de toute restriction. Enfin, en réponse aux réclamations qui lui étaient faites concernant l'insuffisance du montant de $\$ 60,000$, Mercier affirma qu'il était prêt à majorer l'octroi si les statistiques démographiques le justifiaient. "Le gouvernement désire rendre toujours justice et donner entière satisfaction à la minorité protestante de cette province, chaque fois que cela est possible", concluait-il.,75

Les protestants ne tardèrent pas à montrer qu'ils appréciaient la politique de conciliation de Mercier. Le comité protestant remit d'abord son appel, puis l'annula en vertu de l'article 93 de l'AANB. ${ }^{76}$ Et lorsque la Quebec Equal Rights Association intervint dans l'élection provinciale complémentaire $\mathrm{du}$ comté de Brome, les électeurs protestants votèrent pour un conservateur plutôt que pour un adversaire du candidat libéral appartenant à l'ERA. ${ }^{77}$ Moins d'un mois plus tard, lorsque Colby,

73 Documents Relatifs au Règlement de la Question des Biens des Jésuites, 1888-1890 (Québec, s.d.), 56; The Herald, Montréal, 6 nov. 1889. 74 Québec, Sessional Papers (No 5 ) 1890, 24, Sommaire des procédés, 25 sept. 1889 : 335-338.

75 Lettre de Mercier au Très Révérend James Williams, Lord Evêque de Québec, non datée, mais présentée lors de la réunion du Comité protestant du Conseil de l'Instruction publique, le 6 novembre 1889 . Voir Procèsverbaux des Comités catholique et protestant du Conseil de l'Instruction publique dans Documents de la Session, no 5, XXIV (1890): 342-348.

76 The Gazette, Montréal, 15 oct. 1889.

77 Ibid., 21,22 et 29 nov. 1889. 
nouvellement nommé ministre du cabinet, dut se présenter devant l'électorat de Stanstead, les résultats furent encore plus révélateurs. Le comté n'avait suscité aucun candidat de l'ERA pour faire opposition à Colby; mais la section montréalaise s'opposa à l'élection sans opposition de celui qui avait soutenu que les protestants du Québec ne nourrissaient aucun grief contre l'administration Mercier. Malheureusement, leur candidat, Benjamin LeBarron, un marchand à la retraite, mena une très piètre campagne électorale, ne se montrant nullement de taille à lutter contre Colby, en dépit de l'appui des prohibitionnistes. La popularité du nouveau ministre, le refus des libéraux de présenter un candidat, l'habileté avec laquelle Mercier manœuvra face aux protestants, l'appui discret qu'il donna à Colby, tout cela concourut à amener la déconfiture électorale du candidat de l'ERA. Colby remporta trois fois plus de suffrages que l'infortuné LeBarron. ${ }^{78}$

Les résultats obtenus dans Stanstead et Brome encouragèrent le premier ministre à continuer sa politique de conciliation. Quand s'ouvrit la session législative de 1890, Mercier tint parole envers le comité protestant du Conseil de l'Instruction publique au sujet des modifications à apporter à la loi sur les biens des Jésuites. On présenta un amendement visant à modifier les clauses que les protestants jugeaient inacceptables. Le nouveau projet de loi stipulait clairement que le règlement antérieur ne devait pas être interprété comme affectant de quelque façon que ce soit les fonds réservés à l'éducation supérieure - le "cancelled trust" qui existait avant 1888; de plus, il portait la somme accordée aux protestants de $\$ 60,000$ à $\$ 62,961$. Mercier refusa de céder aux pressions individuelles de certains députés protestants qui voulaient lui faire révoquer le préambule de la loi de 1888, mais il donna satisfaction aux revendications du comité protestant. ${ }^{79}$ Le premier ministre fit encore preuve de bonne volonté envers la minorité protestante quand la fameuse question du "B.A.Bill" revint en Chambre. Il semble qu'au printemps de 1889, Mercier avait promis de se montrer favorable à ce projet de loi à la session suivante. ${ }^{80}$ S'il en fut ainsi, il tint

78 Ibid., 12 déc. 1889 ; The Herald, Montréal, 4, 12, 17 et 19 déc. 1889; Toronto Daily Mail, 19 déc. 1889.

79 Université McGill, Archives, Fonds Dawson, Accession 927 [Fonds Dawson (927) ], paquet 52, J. S. Hall à W. Dawson, 21 janv. 1890. Le nouvel Acte était Québec, 53 Victoria, c 31. Sessional Papers ( $N^{\circ} 5$ ) 1890, 24, Sommaire des procédés, 7 mai 1890: 354 .

80 Québec, Sessional Papers ( $N^{\circ} 5$ ) 1889, 23, Sommaire des procédés, 14 mai 1889: 395 . 
parole en 1890. Avant la session, alors que le succès du projet de loi semblait peu probable, ses promoteurs protestants firent appel à Mercier ainsi qu'à Macdonald à Ottawa, cherchant à s'assurer les quelques votes qui permettraient au projet de loi de passer au Conseil législatif de Québec.81 Il se trouva que ce fut moins grâce à l'appui de Mercier et de Macdonald qu'à l'appui de l'Université Laval que le Conseil législatif l'approuva enfin par un vote de dix contre sept. ${ }^{82}$ Mais Mercier n'en avait pas moins usé au mieux de son influence et avait, par le fait même, encouru la colère de quelques-uns de ses partisans nationalistes. ${ }^{83}$ Il se mérita, comme il se devait, l'approbation de nombreux journaux de langue anglaise tels la Sherbrooke Gazette qui était d'avis que "Mr. Mercier deserves thanks of all true lovers of equal rights". ${ }^{84}$ L'Equal Rights Association de Montréal n'était pas nécessairement prête à acclamer Honoré Mercier comme membre honoraire pour la seule raison qu'il avait appuyé le "B.A.Bill", mais, fait très révélateur, lorsque la section de Montréal tint sa réunion d'affaires moins d'une semaine après le vote de la mesure en Chambre, les membres de l'ERA trouvèrent très peu à redire à la conduite du premier ministre. ${ }^{85}$

La politique de conciliation de Mercier eut pour effet de calmer les craintes des protestants et d'affaiblir au Québec le mouvement de l'ERA. Dès janvier, leurs adeptes à Montréal commencèrent à sentir une forte opposition aux efforts qu'ils faisaient pour gagner à leur cause les habitants des Cantons de l'Est. ${ }^{86}$ Quand l'ERA demanda aux Commissions des écoles protestantes de résister au Comité protestant du Conseil de l'Instruction publique qui avait décidé d'accepter les fonds octroyés, les commissaires protestants opposèrent une totale indifférence. ${ }^{87}$ Les élections provinciales de juin témoignèrent clairement avec quelle rapidité déclinait l'Equal Rights Association. Au moment où la lutte électorale commença à devenir

81 Fonds Macdonald, 482: 239950, J. S. Hall à Macdonald, 4 fév. 1890; ibid.: 240098-240099, J. S. Hall à Macdonald, 8 fév. 1890; Fonds Dawson (RBR), boîte "Letters 1889-1890", enveloppe "Letters 1890", R. W. Heneker à W. Dawson, 27 janv. 1890.

82 The Gazette, Montréal, 14 fév. 1890; Fonds Dawson (927), paquet

52, J. S. Hall à W. Dawson, 21 fév. 1890 ; Fonds Macdonald, 482: 240098240099 , J. S. Hall à Macdonald, 8 fév. 1890 .

83 The Gazette, Montréal, 18 fév. 1890.

84 Dans The Herald, Montréal, 15 fév., 1890; aussi Richmond Guardian,

dans The Herald, 10 fév. 1890; The Gazette, Montréal, 14 fév. 1890.

85 The Herald, Montréal, 19 fév. 1890.

86 The Gazette, Montréal, 8 jan. 1890.

87 Ibid., 12 mai 1890. 
plus serrée, les Equal Righters publièrent un manifeste destiné à guider l'électorat. Les premier et dernier points du manifeste résument bien le document: "1. Complete separation of church and state", et "10. Country before party". Entre ces deux points, le document faisait des suggestions sur les octrois aux groupes religieux, la dîme, la prohibition du commerce des spiritueux et la "promotion of legislation tending to improve the condition of the working classes". ${ }^{88}$ Le fait que le document ne préconisait aucun moyen d'atteindre ces objectifs indiquait bien l'inefficacité de l'ERA du Québec. Soulignons que la loi sur les biens des Jésuites ne figura pas parmi les thèmes majeurs de la campagne.

Les électeurs protestants manifestèrent leur indifférence en refusant leur appui aux adversaires de Mercier. Déjà paralysés par leur organisation inadéquate et par leur leadership inefficace en dehors de Montréal ${ }^{89}$, les conservateurs se rendirent désormais à l'évidence qu'ils ne pouvaient pas compter sur les riches protestants de langue anglaise comme bailleurs de fonds électoraux. Bien que Macdonald eût escompté que la piètre politique financière de Mercier favoriserait les Bleus ${ }^{90}$, les "favorites of Plutus", comme Chapleau appelait les hommes d'affaires et les industriels de Montréal ${ }^{91}$, n'étaient pas convaincus qu'il serait dans leur intérêt de s'opposer à Mercier. Alors que des entreprises industrielles, commerciales et de transport demeuraient neutres, ${ }^{92}$ la compagnie du Grand Tronc, elle, accordait ouvertement son appui aux libéraux pour rappeler à Macdonald qu'il était trop favorable à leur rivale, le Pacifique Canadien. ${ }^{93}$ Selon toute apparence, les hommes d'affaires étaient convaincus que "both parties are equally responsible for the tax on the English speaking population and it is difficult to get people to believe that either party will relax the grasp upon our pockets". ${ }^{94}$ Ayant toujours en mémoire les années de corruption et de mauvaise administration sous les Bleus, ils ne prenaient pas au sérieux les accusations des conservateurs qui soutenaient que Mercier était en train de mettre l'économie en péril et d'augmenter dangereusement la dette provinciale. ${ }^{95}$ Sir George Stephen

88 Ibid., 2 juin 1890.

89 Miller, "Impact of the Jesuits' Estates Act", 357.

90 Fonds Macdonald, 529, partie II: 490-491, Macdonald à J. J. C. Abbott, 7 juin 1890 .

91 Ibid., 205: 87343-87346, J. A. Chapleau à Macdonald, 13 juin 1890. 92 Ibid., 186: 77281-77283, J. J. C. Abbott à Macdonald, 9 juin 1890. 93 Ibid., 485: 242469-242471, R. White à Macdonald, 28 mai 1890. 94 Ibid.

95 The Herald, Montréal, mai-juin 1890, passim. 
comprenait l'attitude cynique des protestants anglophones qui affirmait que "all French are "tarred with one stick" et que "therefore Mercier is about as good as the average"96. Alors que le premier ministre Macdonald avait espéré des hommes d'affaires de Montréal une contribution allant de $\$ 50,000$ à $\$ 100,000$ pour renverser Mercier, il en reçut moins de $\$ 20,000{ }^{97}$; alors que les conservateurs et les Equal Righters avaient espéré que les protestants renverseraient Mercier, les Rouges balayèrent la province. Les libéraux prirent quarante-cinq des soixantetrois sièges, ce qui constituait un progrès spectaculaire par rapport à la victoire obtenue de justesse en $1886 .{ }^{98}$ Non seulement cette élection affermait-elle l'emprise de Mercier sur la province, mais elle donnait le coup de grâce aux Equal Righters du Québec. ${ }^{99}$

\section{IV}

Pourquoi la loi sur les biens des Jésuites avait-elle constitué un problème majeur au Québec ? Le premier ministre Macdonald croyait que si Mercier avait délibérément ajouté à la loi un préambule si provocateur pour la population protestante, c'était dans l'intention de fomenter des dissensions entre Ottawa et Québec qui serviraient ses propres fins politiques. Rien, cependant, ni dans le contexte ni dans la lettre de la loi ne justifiait un tel soupçon. Le problème des biens des Jésuites en était un très ancien qui demandait à être solutionné et Mercier avait mené cette affaire délicate avec un tact remarquable. S'il avait inclus dans le préambule la correspondance avec le Saint-Siège, ce n'était pas pour inciter les protestants à la colère, mais, ainsi qu'il l'expliquait lui-même, pour montrer clairement que le pacte avait été conclu avec l'approbation et la bénédiction du Pape. S'il avait soumis au Vatican le partage qu'il entendait faire des biens des Jésuites, ce n'était pas pour insulter les Orangistes

96 Fonds Macdonald, 272: 124429-124436, G. Stephen à Macdonald, 6 juin 1890; aussi ibid., 486: 243018-243019, W. Graeme à Macdonald, 24 juin 1890.

97 Ibid., 529, partie II : 490-491, Macdonald à J. J. C. Abbott, 7 juin 1890 ; ibid., 205: 87343-87346, J. A. Chapleau à Macdonald, 13 juin 1890; ibid., 486: 242782-242783, R. White à Macdonald, 13 juin 1890.

98 Cette analyse est fondée sur J. A. Gemmill, The Canadian Parliamentary Companion 1891 (Ottawa, 1891), 260-280. Il n'est pas d'accord avec les résultats dans J. Hamelin, J. Letarte, et M. Hamelin, "Les élections provinciales dans le Québec", Cahiers de Géographie de Québec, IV (19591960): 24: libéraux et nationaux, 47; conservateurs, 26. 25 nov. 1890. 
mais pour éviter toute querelle entre les bénéficiaires catholiques. L'agitation contre la loi qui avait suivi n'était évidemment pas imputable à Mercier; elle ne tenait pas non plus à quelque défaut inhérent à la loi elle-même.

Mais la loi sur les biens des Jésuites n'avait pas moins provoqué les protestants. Dès le début du débat à l'Assemblée législative en 1888, les députés protestants s'en étaient montrés insatisfaits, même s'ils n'eurent pas le courage de s'y opposer par vote. A l'été de 1888, plusieurs journaux du Québec avaient élevé la voix contre la loi, et ce fut une organisation protestante du Québec, le Montreal Presbytery, qui avait été la première à suggérer l'intervention $d u$ gouvernement fédéral dans cette affaire. Les protestants du Québec avaient considéré cette loi comme le symbole d'une puissance qu'ils n'aimaient pas et dont ils redoutaient l'influence grandissante dans la province: l'ultramontanisme et la politique nationaliste de Mercier. Faisant suite aux lois sur la conversion de la dette et sur l'incorporation des compagnies, la loi sur les biens des Jésuites était apparue à de nombreux protestants comme faisant partie d'une conspiration contre eux. ${ }^{100}$ C'était, en effet, le signe d'un changement dans la vie québécoise où la communauté canadienne-française s'affirmait de plus en plus sous la direction d'hommes qui proclamaient ardemment leur catholicisme et leur fierté canadienne-française. Une revue anglaise avait bien capté l'inquiétude de la minorité quand elle prédit que "a little more of such legislation... is needed to complete the new Conquest. The process of extirpating British ideas and institutions is going on apace in this uniquely constituted province; and it has made greater strides under the few months of Mr. Mercier's rule than for the whole period covered by the era of responsible government." "101 Pour les protestants du Québec, la loi sur les biens des Jésuites était le signe d'un Québec en voie de détérioration et s'orientant vers cette tragédie que Robert Sellar déplorera une génération plus tard. ${ }^{102}$

Compte tenu que la minorité religieuse considérait cette loi comme un symptôme de dégénérescence, un avertissement le long d'un sentier non encore entièrement frayé, sa réaction a été

100 Fonds Dawson (927), paquet 52, Memo sur Question des Biens des Jésuites, 8 juin 1889, par Principal Dawson; Fonds Dawson (RBR), boîte "Letters 1887-1889", enveloppe "Letters 1889", R. Dawson à W. Dawson, mars 1889.

101 Richmond Guardian, cité par le Canadian Gleaner, 27 juin 1888.

102 R. Sellar, The Tragedy of Quebec: the Expulsion of its Protestant Farmers (Huntingdon, 1907). 
timide et dénuée de réalisme. Se sentant seule et isolée, elle n'avait d'abord presque rien fait pour combattre le projet de loi. ${ }^{103}$ Mais dès que les Ontariens eurent manifesté de l'inquiétude, les protestants du Québec emboîtèrent le pas avec empressement à leurs coréligionnaires de l'Ouest et les suivirent servilement. Ils furent ainsi entraînés dans le sillage d'une campagne à la fois importante et plus agressive. Aussi, quand leurs frères ontariens firent preuve de peu d'intérêt à l'endroit de leurs objectifs lors de la convention anti-jésuites, les membres de la Quebec Equal Rights Association s'étaient sentis abandonnés. A partir de ce moment, leur action avait été fortement marquée par ce pessimisme qui aboutit à une ineptie complète durant les élections provinciales de 1890.

Le mouvement du Quebec Equal Rights se désintégra non seulement parce qu'il avait été trahi par l'Ontario mais encore parce qu'Honoré Mercier avait su manœuvrer en maître. Bien que le premier ministre eût été piqué au vif par l'agitation qui s'était manifestée alors, il avait accédé à presque toutes les demandes de révision de la loi que lui avaient faites les protestants, et il avait fait preuve de bonne volonté jusqu'à accorder son appui à une mesure qui était chère au cœur de la minorité religieuse, le "B.A.Bill". Les habiles tactiques de Mercier ainsi que le fait inhibant d'avoir à lutter sans l'appui des protestants ontariens avaient produit leur effet aux élections de 1889 et de 1890. Durant les élections provinciales de 1890, Mercier montra qu'il avait surmonté en grande partie l'hostilité protestante et qu'il avait étouffé l'agitation soulevée contre son projet de loi relative au règlement de la question des biens des Jésuites.

Traduction de JULIETte VeILleuX

Département d'anglais

Université de Sherbrooke

103 APC, Fonds G. M. Grant, 7: 2400A-2400C, S. E. Dawson à G. M. Grant, 7 mars 1889. 